\title{
Above 1 ATM Gas Reactions of Nanocatalysts in a Conventional TEM
}

\author{
Huolin L. Xin, * and Haimei Zheng* \\ *Materials Sciences Division, Lawrence Berkeley National Laboratory, Berkeley, CA 94720
}

Our understanding of catalyst-gas interactions has been greatly advanced over the past decade on account of the availability of transmission electron microscopes (TEMs) equipped with differentially pumped environmental cells. The operational pressures in these differentially pumped environmental TEM (DP-ETEM) instruments, however, are limited to 10-20 mbar due to the long gas path length. Yet, many industrial reactions are operated at pressures equal or higher than 1 bar100 times higher than that in the DP-ETEM. This drawback of DP-ETEM has rendered our understating of catalyst behavior incomplete. Here, we present a membrane enclosed gas flow cell that allows a differential pressure up to 3 bar. This gas flow cell coupled with silicon carbide membrane heaters allows for in situ heating of solid materials from $95{ }^{\circ} \mathrm{C}$ to $400{ }^{\circ} \mathrm{C}$ in flowing reactive gases. We demonstrate that in conventional thermionic TEM instruments, atomic fringe resolution in this closed environmental system can be preserved with and without flowing gases. We present in situ oxidation of cobalt nanocatalyts that reveals real-time dynamics of the Kirkendall effect.

Fig. 1 shows the flow holder system. The tip, highlighted by a dashed box in Fig. 1c, is designed to be detachable. Gases are injected into the guided channel through the red PEEK tubing (Fig. 1c-inset). TEM gas cell (Fig. 1d) is composed of two silicon chips-a spacing chip and a regular chip. The chips are fabricated to be $3 \mathrm{~mm} \times 3 \mathrm{~mm}$ in size with $50 \mu \mathrm{m} \times 200 \mu \mathrm{m}$ windows. The windows are covered with 25-50 nm thick silicon nitride membranes. When heating is needed, a $\mathrm{SiC}_{\mathrm{x}}$ membrane-type heater chip is used. The heating element is a conductive $\mathrm{SiC}_{\mathrm{x}}$ membrane that is deposited on the $\mathrm{SiN}_{\mathrm{x}}$ sample supporting membrane (Fig. 1e).

With differential pressure applied, the $\mathrm{SiN}_{\mathrm{x}}$ membranes in the windows will bulge out increasing the gas path length. We found that, with a window width of $60 \mu \mathrm{m}$, the maximum displacement of the membrane approaches $1 \mu \mathrm{m}$ (Fig. 2a). However, in our $\mathrm{LaB}_{6}$ instrument, the information transfer of the amorphous membrane remained unchanged with and without static atmospheric gases (Fig. 2c,d). This is due to the fact a micron thick of gas is only equivalent to $1 \mathrm{~nm}$ of solid/liquid materials. Compared to the $25-50 \mathrm{~nm}$ thick SiNx membranes, the $\sim 2$ micron gas path length results in ignorable degradation of resolution in our $\mathrm{LaB}_{6}$ instrument. However, if gas tubing were attached to the holder and especially if gas were flowing, coupled vibrations could limit information transfer. Fig. $2 \mathrm{e}$ and $2 \mathrm{f}$ show that 2.2-angstrom fringes can be resolved in a static atmosphere and in oxygen gas with a flow rate of $0.2 \mathrm{sccs}$. It demonstrates high resolution capability of our flow holder system under realistic environmental conditions.

We operated an oxidation reaction of cobalt nanoparticles in our gas cell with temperature ramping from $150{ }^{\circ} \mathrm{C}$ to $250{ }^{\circ} \mathrm{C}$ at $\sim 5^{\circ} \mathrm{C} / \mathrm{sec}$. Fig. 3a shows the time dependent drift rate as a function of time. The relatively slow drift $(\max <2.9 \mathrm{~nm} / \mathrm{sec})$, and the short settling time constant $(75$ $\mathrm{sec}$ ) with relatively fast temperature ramping are attributed to the membrane-type heater design. It provides in situ tracking and imaging capabilities improved over traditional furnace-type heating holders. Fig. $3 b$ shows the time-lapse still image of three selected particles. Fig. $3 b(I)$ and $3 b(I I)$ show, due to spatially anisotropic bridge diffusion, the cores shrunk with a unidirectional retraction front (Fig. 3b(I)) and a sweeping retraction front (Fig. 3b(II)) respectively. Fig. 3b(III) visualizes the shrinkage of the core in projection view - the retraction front's normal direction is close to the beam incident direction. It shows that in projection, the residual metallic puddle was relatively round following the shape of the hollow shell surface. [1]

\section{References}


[1] Supported by the Office of Basic Energy Sciences, Chemical Science Division of the U.S. DOE under Contrast No. DE-AC02-05CH11231. H Zheng thanks the funding support from DOE Early Career Research Program.
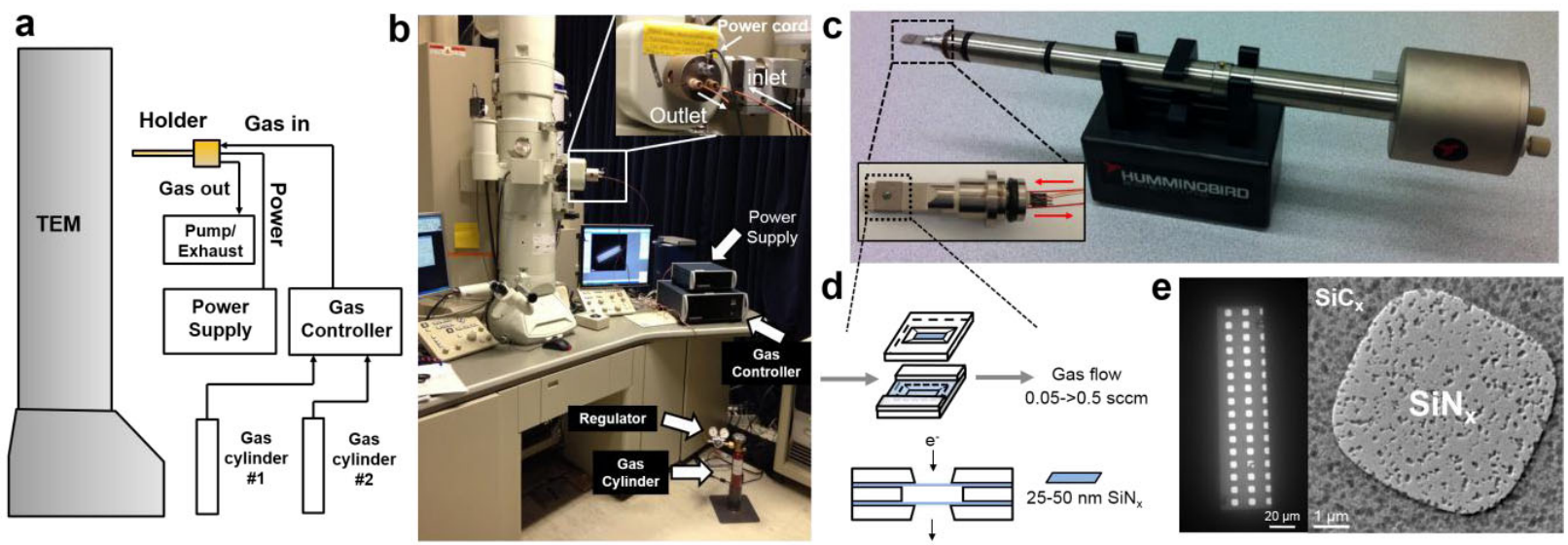

Fig. 1. (a-b) The gas flow holder system. (c) The gas-flow heating holder. (d) The MEMS-type gas flow cell. (e) The electron transparent windows of the $\mathrm{SiC}_{\mathrm{x}}$ membrane-type heating cell.
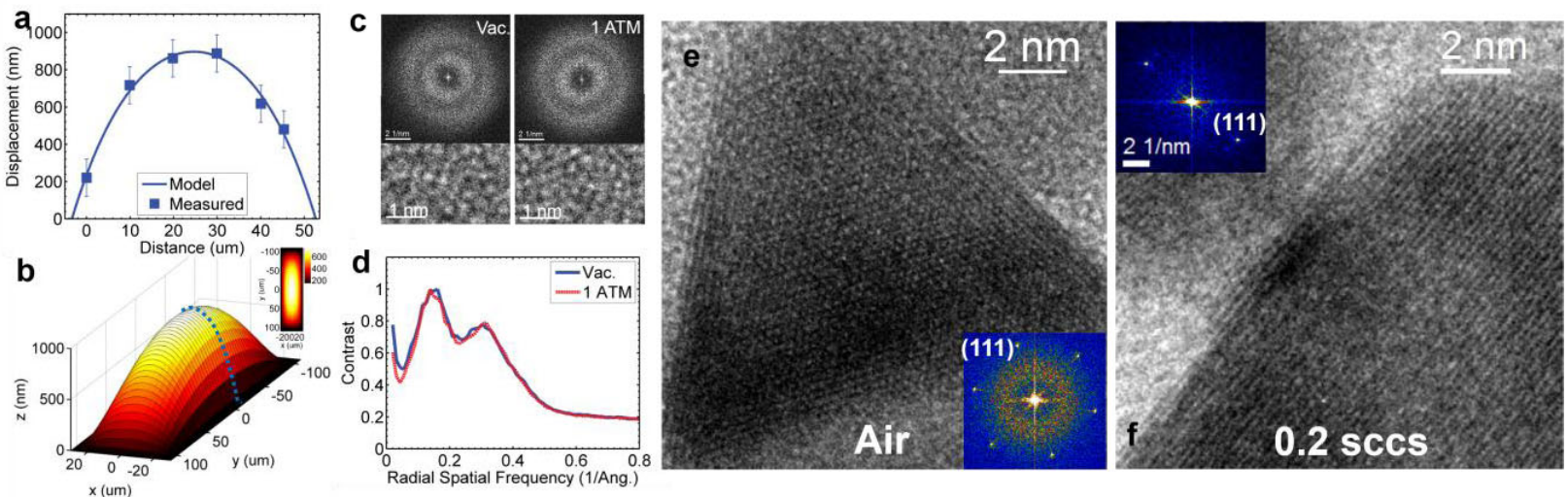

Fig. 2. $\mathrm{SiN}_{\mathrm{x}}$ window bulging and imaging resolution. (a) The measured membrane buldging displacement. (b) The two buldging profile of the window membrane. (c-d) The diffratogram and the images of the amorphous membrane w/ and w/o gas in the cell. (e-f) Imaging of Pd particles in (e) static atmospheric air and in (f) flow oxygen at 0.2 standard cubic centimeter per second (sccs).
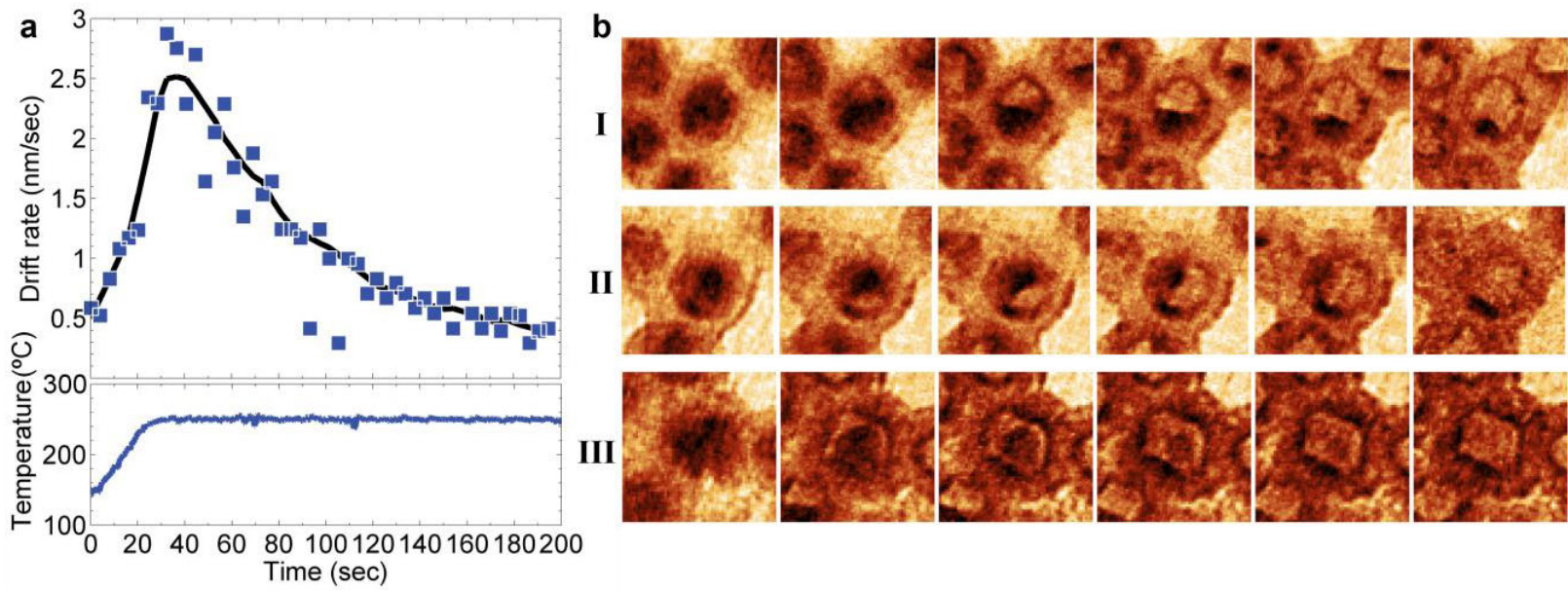

III

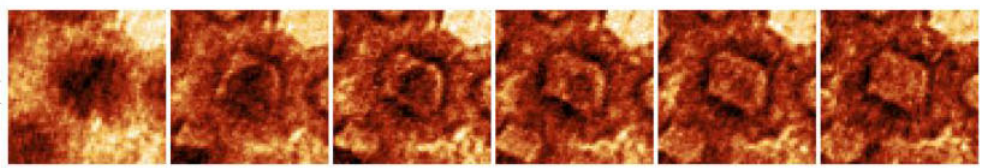

Fig. 3. In situ heating of cobalt nanoparticles in flowing oxygen. (a) Measured drift rate as a function of time. (b) Time lapse images showing the oxidation dynamics of cobalt nanoparticles. 